International Journal of Pure and Applied Mathematics

Volume 115 No. 3 2017, 549-560

ISSN: 1311-8080 (printed version); ISSN: 1314-3395 (on-line version)

url: http://www.ijpam.eu

doi: 10.12732 /ijpam.v115i3.9

\title{
ON THE EXISTENCE FOR ALMOST EVERYWHERE SOLUTION OF MULTI-DIMENSIONAL MIXED PROBLEM FOR ONE CLASS THIRD ORDER DIFFERENTIAL EQUATIONS WITH NONLINEAR OPERATOR IN THE RIGHT-HAND SIDE
}

\author{
Samad Aliyev ${ }^{1}$, Arzu Aliyeva ${ }^{2}$ \\ ${ }^{1}$ Department of Mathematics and Methods of its Teaching \\ Faculty of Mechanics and Mathematics \\ Baku State University \\ AZ 1148, 23 Z. Khalilov Street, Baku, REPUBLIC OF AZERBAIJAN \\ ${ }^{2}$ Department of Differential Equations \\ Institute of Mathematics and Mechanics \\ National Academy of Sciences of Azerbaijan \\ AZ 1141, 9 B.Vagabzade Street, Baku, REPUBLIC OF AZERBAIJAN
}

\begin{abstract}
The existence theorem for the solution of an almost everywhere multidimensional mixed problem for a class of third-order differential equations with nonlinear operator righthand side has been proved.
\end{abstract}

AMS Subject Classification: 35A01, 35A08, 35A22

Key Words: continuously differentiable finite functions, differential equations, Fourier method

\section{Introduction}

In this paper we study the existence of a solution almost everywhere of the

Received: $\quad$ May 29, 2017

Revised: $\quad$ July 2, 2017

Published: July 27, 2017

${ }^{\S}$ Correspondence author (c) 2017 Academic Publications, Ltd. url: www.acadpubl.eu 
following multidimensional mixed problem:

$$
\begin{gathered}
\frac{\partial^{2} u(t, x)}{\partial t^{2}}-\frac{\partial}{\partial t} L(u(t, x))=\mathcal{F}(u(t, x)) \quad(t \in[0, T], x \in \Omega), \\
u(0, x)=\varphi(x) \quad(x \in \Omega), \quad u_{t}(0, x)=\psi(x) \quad(x \in \Omega), \\
\left.u(t, x)\right|_{\Gamma}=0,
\end{gathered}
$$

where $0<T<+\infty ; x=\left(x_{1}, \ldots, x_{n}\right), \Omega-n$ - dimensional bounded domain with a sufficiently smooth boundary $S, \Gamma=[0, T] \times S$;

$$
L(u(t, x))=\sum_{i, j=1}^{n} \frac{\partial}{\partial x_{i}}\left(a_{i j}(x) \frac{\partial u(t, x)}{\partial x_{j}}\right)-a(x) u(t, x),
$$

and the functions $a_{i j}(x)(i, j=\overline{1, n}) \quad a(x)$ are measurable and bounded in $\Omega$ and in the domain $\Omega$ satisfy the conditions

$$
a_{i j}(x)=a_{j i}(x), a(x) \geq 0, \sum_{i, j=1}^{n} a_{i j}(x) \xi_{i} \xi_{j} \geq \alpha \cdot \sum_{i=1}^{n} \xi_{i}^{2} \quad(\alpha=\text { const }>0),
$$

$\xi_{i}-$ are any real numbers; $\varphi, \psi-$ the specified functions; $\mathcal{F}$ - is, in general, a nonlinear operator, $u(t, x)$ - is the desired function.

In investigating the almost everywhere solution of problem (1) - (3), we will use the classes of functions $\stackrel{\circ}{\mathcal{D}}$ and $\stackrel{\circ}{\mathcal{D}}_{1}$, Introduced by K. Friedrichs (see $[1, \mathrm{p}$. $38])$.

\section{The Main Definitions and Notations}

The closure of the set of all continuously differentiable finite functions in $\Omega$ in the norm $W_{2}^{1}(\Omega)$ is called the class $\stackrel{\circ}{\mathcal{D}}(\Omega)$. It is obvious that $\stackrel{\circ}{\mathcal{D}}(\Omega) \subset W_{2}^{1}(\Omega)$.

Denote by $\dot{\mathcal{D}}_{1}\left(Q_{T}\right)\left(Q_{T} \equiv[0, T] \times \Omega\right)$ totality of all continuously differentiable functions are equal to zero in the $\delta$ - neighbourhood of the lateral surface of the cylinder $Q_{T}$, having the form: $Q_{T, \delta}=[0, T] \times \Omega_{\delta}$, where $\Omega_{\delta}$ is the collection of points $\Omega$, removed from the boundary $\Omega$ by a distance not exceeding $\delta$. The closure $\dot{\mathcal{D}}_{1}\left(Q_{T}\right)$ in the norm $W_{2}^{1}\left(Q_{T}\right)$ is denoted by $\stackrel{\circ}{\mathcal{D}}_{1}\left(Q_{T}\right)$. Obviously, $\stackrel{\circ}{\mathcal{D}}_{1}\left(Q_{T}\right) \subset W_{2}^{1}\left(Q_{T}\right)$.

Definition. The function $u(t, x) \in \stackrel{\circ}{\mathcal{D}}_{1}\left(Q_{T}\right)$, belonging to the space $L_{2}\left(Q_{T}\right)$ together with all its derivatives $u_{t}(t, x), u_{x_{i}}(t, x)(i=\overline{1, n}), u_{t x_{i}}(t, x)(i=\overline{1, n})$, 
$u_{x_{i} x_{j}}(t, x)(i, j=\overline{1, n}), u_{t t}(t, x), u_{t x_{i} x_{j}}(t, x)(i, j=\overline{1, n})$, satisfying equation (1) almost everywhere in $Q_{T}$ and taking initial values (2) almost everywhere in $\Omega$ is called a solution almost everywhere of the problem (1) - (3).

It should be noted that many problems in the theory of elasticity, in particular, the problem of longitudinal vibration of an elastic-viscous inhomogeneous rod, the problem of longitudinal impact by an absolutely rigid body in an elastic-viscous inhomogeneous rod of finite length and variable cross section, the propagation of waves in a viscoelastic body, propagation pulses along nerve axons (neurons), etc., reduce to the solution of mixed problems for various particular cases of equation (1).

In the beginning, we will note some papers related to problem (1) - (3).

In [2] a mixed problem for the equation below was considered

$$
\begin{aligned}
u_{t t}(t, x)- & \alpha \Delta u(t, x)-\Delta u_{t}(t, x) \\
& =f\left(t, x, u(t, x), u_{t}(t, x), \mathcal{D} u(t, x), \mathcal{D} u_{t}(t, x), \mathcal{D}^{2} u(t, x), \mathcal{D}^{2} u_{t}(t, x)\right)
\end{aligned}
$$

Under certain special conditions with respect to the nonlinear function $f$ the existence of the solution of the problem under consideration for all $t>0$ has been proved.

In [3], a mixed problem for the equation

$$
u_{t t}(t, x)-\alpha \Delta u_{t}(t, x)-\Delta u(t, x)=f(t, x, u(t, x))
$$

with a nonlinearity of the type $|u|^{p-1} \cdot u$ was considered. The conditions for the existence of a global weak solution of this problem are indicated.

Further, in [4] a special case of problem (1) - (3) is considered, when the operator $\mathcal{F}$, appearing on the right-hand side of equation (1), is an operator of the type of a function generated by the function $f\left(t, x, u, u_{t}, u_{x}, u_{t x}, u_{x x}\right)$; the question of the existence and uniqueness of the solution almost everywhere of problem (1) - (3) is investigated, namely, by combining the generalized principle of compressed maps with the Schauder principle for any dimensions $n$ the existence theorem for small (that is, valid for sufficiently small values of $T$ ) and uniqueness as a whole (that is, valid for any finite value $T$ ) of the solution almost everywhere of the problem (1) - (3)) was proved, and using the method of a priori estimates for all dimensions $n$ the existence theorem on the whole of the solution of problem (1) - (3) almost everywhere was proved. 


\section{Results and Discussion}

We will consider some well-known facts and will add a number of new auxiliary facts in order to investigate the solutions of problem almost everywhere (1) (3).

1. As is known, the operator $L$, generated by the differential expression (4) and the boundary condition (3), has a countable system of negative eigenvalues

$$
0>-\lambda_{1}^{2} \geq-\lambda_{2}^{2} \geq \ldots \geq-\lambda_{s}^{2} \geq \ldots \quad\left(0<\lambda_{s} \rightarrow+\infty \text { as } s \rightarrow \infty\right)
$$

nd the corresponding complete orthonormal in $L_{2}(\Omega)$ system of generalized eigenfunctions $v_{s}(x)$, and by the generalized eigenfunction $v_{s}(x)$ of the operator $L$ we mean a function $v_{s}(x)$ that is not identically zero, which belongs to the class $\stackrel{\circ}{\mathcal{D}}(\Omega)$ and for any function $\Phi(x)$ from $\stackrel{\circ}{\mathcal{D}}(\Omega)$ satisfies the integral identity.

$$
\int_{\Omega}\left\{\sum_{i, j=1}^{n} a_{i j}(x) \frac{\partial v_{s}(x)}{\partial x_{i}} \cdot \frac{\partial \Phi(x)}{\partial x_{j}}+a(x) v_{s}(x) \Phi(x)\right\} d x=\lambda_{s}^{2} \int_{\Omega} v_{s}(x) \Phi(x) d x .
$$

It is obvious that every solution almost everywhere of problem (1) - (3) has the form:

$$
u(t, x)=\sum_{s=1}^{\infty} u_{s}(t) v_{s}(x),
$$

where $u_{s}(t)=\int_{\Omega} u(t, x) v_{s}(x) d x(s=1,2, \ldots)$. Then, after applying the formal scheme of the Fourier method, finding the Fourier coefficients $u_{s}(t)$ of the desired solution almost everywhere $u(t, x)$ of problem (1)-(3) reduces to solving the following countable system of nonlinear integro-differential equations:

$$
\begin{array}{r}
u_{s}(t)=\varphi_{s}+\frac{1}{\lambda_{s}^{2}}\left(1-e^{-\lambda_{s}^{2} t}\right) \psi_{s}+\frac{1}{\lambda_{s}^{2}} \int_{0}^{t} \int_{\Omega} \mathcal{F}(u(\tau, x))\left[1-e^{-\lambda_{s}^{2}(t-\tau)}\right] v_{s}(x) d x d \tau \\
(s=1,2, \ldots ; t \in[0, T]), \quad(6)
\end{array}
$$

where

$$
\varphi_{s}=\int_{\Omega} \varphi(x) v_{s}(x) d x, \quad \psi_{s}=\int_{\Omega} \psi(x) v_{s}(x) d x .
$$

2. Proceeding from the definition of a solution almost everywhere of problem (1) - (3), the following lemma is easily proved. 
Lemma. If $u(t, x)$ is a solution almost everywhere of problem (1) - (3) and generalized derivatives $\frac{\partial}{\partial x_{k}} a_{i j}(x)(i, j, k=\overline{1, n})$ are bounded in $\Omega$, then the functions $u_{s}(t)(s=1,2, \ldots)$ satisfy the system $(6)$ on $[0, T]$.

3. We denote by $B_{\beta_{0}, \ldots, \beta_{l}, T}^{\alpha_{0}, \ldots, \alpha_{l}}$ the set of all functions $u(t, x)$ of the form (5), which are considered in $[0, T] \times \Omega$ and for which all the functions $u_{s}(t) \in$ $C^{(l)}([0, T])$ and

$$
J_{T}(u) \equiv \sum_{i=0}^{l}\left\{\sum_{s=1}^{\infty}\left(\lambda_{s}^{\alpha_{i}} \cdot \max _{0 \leq t \leq T}\left|u_{s}^{(i)}(t)\right|\right)^{\beta_{i}}\right\}^{1 / \beta_{i}}<+\infty
$$

where $\alpha_{i} \geq 0,1 \leq \beta_{i} \leq 2(i=\overline{0, l})$. The norm in this set is defined as follows: $\|u\|=J_{T}(u)$. Obviously, all these spaces are Banach spaces (see [5, p. 50])

4. We use the following notation:

$$
\begin{aligned}
\mathcal{D}(u(t, x), V(t, x)) \equiv \int_{\Omega}\left[\sum_{i, j=1}^{n} a_{i j}(x) \frac{\partial u(t, x)}{\partial x_{i}} \frac{\partial V(t, x)}{\partial x_{j}}\right. & +a(x) u(t, x) V(t, x)] d x, \\
\mathcal{D}(u(t, x), u(t, x)) \equiv & \mathcal{D}(u(t, x)) \\
& =\int_{\Omega}\left[\sum_{i, j=1}^{n} a_{i j}(x) \frac{\partial u(t, x)}{\partial x_{i}} \cdot \frac{\partial U(t, x)}{\partial x_{j}}+a(x) u^{2}(t, x)\right]^{d x} .
\end{aligned}
$$

Then it is obvious that for any $s(s=1,2, \ldots)$

$$
\mathcal{D}\left(\frac{v_{s}(x)}{\lambda_{s}}\right)=\frac{1}{\lambda_{s}^{2}} \cdot \mathcal{D}\left(v_{s}(x)\right)=1
$$

Since for any natural number $N$

$$
\begin{aligned}
0 \leq \mathcal{D}(z(t, x)- & \left.\sum_{s=1}^{N} \mathcal{D}\left(z(t, x), \frac{1}{\lambda_{s}} v_{s}(x)\right) \cdot \frac{v_{s}(x)}{\lambda_{s}}\right) \\
& =\mathcal{D}(z(t, x))-2 \sum_{s=1}^{N}\left[\mathcal{D}\left(z(t, x), \frac{1}{\lambda_{s}} v_{s}(x)\right)\right]^{2} \\
& +\sum_{s=1}^{N}\left[\mathcal{D}\left(z(t, x), \frac{1}{\lambda_{s}} v_{s}(x)\right)\right]^{2} \cdot \mathcal{D}\left(\frac{v_{s}(x)}{\lambda_{s}}\right)
\end{aligned}
$$




$$
=\mathcal{D}(z(t, x))-\sum_{s=1}^{N}\left[\mathcal{D}\left(z(t, x), \frac{1}{\lambda_{s}} v_{s}(x)\right)\right]^{2},
$$

then

$$
\sum_{s=1}^{\infty}\left[\mathcal{D}\left(z(t, x), \frac{1}{\lambda_{s}} v_{s}(x)\right)\right]^{2} \leq \mathcal{D}(z(t, x)) .
$$

Similarly to (7), the following inequality is proved:

$$
\sum_{s=1}^{\infty}\left(\lambda_{s} \psi_{s}\right)^{2} \leq \mathcal{D}(\psi(x), \psi(x)) .
$$

Theorem. Let 1. $a_{i j}(x) \in C^{(2)}(\bar{\Omega})(i, j=\overline{1, n}) ; a(x) \in C^{(1)}(\bar{\Omega}) ; S \in$ $C^{3}$; the eigenfunctions $v_{s}(x)$ of the operator $L$ under the boundary condition $\left.v_{s}(x)\right|_{s}=0$ are three times continuously differentiable on $\bar{\Omega} ; \varphi(x) \in W_{2}^{3}(\Omega)$, $\varphi(x), L \varphi(x) \in \stackrel{\circ}{\mathcal{D}}(\Omega) ; \psi(x) \in W_{2}^{2}(\Omega) \cap \stackrel{\circ}{\mathcal{D}}(\Omega)$.

2. The operator $\mathcal{F}$ acts from the ball $\mathcal{K}\left(\|u-W\|_{B_{2, T}^{1}} \leq R\right)$ in $L_{2}\left(Q_{T}\right)$ is continuous and bounded, where $0<R<+\infty$,

$$
W(t, x) \equiv \sum_{s=1}^{\infty}\left\{\varphi_{s}+\frac{1}{\lambda_{s}^{2}}\left[1-e^{-\lambda_{s}^{2} t}\right] \psi_{s}\right\} \cdot v_{s}(x)
$$

3. $\frac{\sqrt{T}}{\lambda_{1}^{2}} \cdot \sup _{u \in \mathcal{K}}\left\{\|\mathcal{F}(u)\|_{L_{2}\left(Q_{T}\right)}\right\} \leq R$.

4. For each $u \in \stackrel{\circ}{\mathcal{D}}_{1}\left(Q_{T}\right), \mathcal{F}(u) \in \stackrel{\circ}{\mathcal{D}}_{1}\left(Q_{T}\right)$.

Then problem (1) - (3) has a solution almost everywhere.

Proof. First we take the following notation:

$$
\begin{gathered}
\mathcal{P}(u(t, x)) \equiv \sum_{s=1}^{\infty} \frac{1}{\lambda_{s}^{2}} \int_{0}^{t} \int_{\Omega} u(\tau, \xi) v_{s}(\xi) \cdot\left[1-e^{-\lambda_{s}^{2}(t-\tau)}\right] d \xi d \tau \cdot v_{s}(x), \\
Q(u(t, x)) \equiv W(t, x)+\mathcal{P} \mathcal{F}(u(t, x)) .
\end{gathered}
$$

It follows from condition 1 of the theorem that $W(t, x) \in B_{2, T}^{1}$. If for each $u(t, x) \in \mathcal{K}$ we denote

$$
\mathcal{P} \mathcal{F}(u(t, x))=\sum_{s=1}^{\infty} \tilde{u}_{s}(t) v_{s}(x)
$$


where

$$
\tilde{u}_{s}(t)=\frac{1}{\lambda_{s}^{2}} \int_{0}^{t} \int_{\Omega} \mathcal{F}(u(\tau, \xi)) v_{s}(\xi) \cdot\left[1-e^{-\lambda_{s}^{2}(t-\tau)}\right] d \xi d \tau,
$$

then it is easy to obtain that for any natural $s, N$ and for any $u \in \mathcal{K}, t \in[0, T]$ :

$$
\begin{gathered}
\left|\tilde{u}_{s}(t)\right| \leq \frac{1}{\lambda_{s}^{2}} \cdot \sqrt{T} \cdot a_{0}, \quad\left|u_{s}^{\prime}(t)\right| \leq \sqrt{T} \cdot a_{0}, \\
\left\{\sum_{s=N}^{\infty}\left(\lambda_{s} \cdot \max _{0 \leq t \leq T}\left|\tilde{u}_{s}(t)\right|\right)^{2}\right\}^{\frac{1}{2}} \leq \frac{\sqrt{T}}{\lambda_{N}^{2}} \cdot\left\{\int_{0}^{T} \int_{\Omega}[\mathcal{F}(u(t, x))]^{2} d x d t\right\}^{\frac{1}{2}} \leq \\
\leq \frac{\sqrt{T}}{\lambda_{N}^{2}} \cdot a_{0},
\end{gathered}
$$

where

$$
a_{0} \equiv \sup _{u \in \mathcal{K}}\left\{\|\mathcal{F}(u)\|_{L_{2}\left(Q_{T}\right)}\right\} .
$$

From the relations (11) and (12), by Theorem 1.1 of $[4$, p. 45] about compactness criteria of sets in spaces $B_{\beta_{0}, \ldots, \beta_{l}, T}^{\alpha_{0} \ldots, \alpha_{l}}$, the compactness of the set $\mathcal{P} \mathcal{F} \mathcal{K}$ $B_{2, T}^{1}$ follows.

Further, for any $u, v \in \mathcal{K}$, we have:

$$
\begin{gathered}
\|Q(u)-W\|_{B_{2, T}^{1}} \leq \frac{\sqrt{T}}{\lambda_{1}^{2}} \cdot\|\mathcal{F}(u)\|_{L_{2}\left(Q_{T}\right)} \leq \frac{\sqrt{T}}{\lambda_{1}^{2}} \cdot a_{0} \leq R, \\
\|Q(u)-Q(v)\|_{B_{2, T}^{1}} \leq \frac{\sqrt{T}}{\lambda_{1}^{2}} \cdot\|\mathcal{F}(u)-\mathcal{F}(v)\|_{L_{2}\left(Q_{T}\right)},
\end{gathered}
$$

where the operator $Q$ is defined by the relation (10).

It is clear from (13) that the operator $Q$ converts the ball $\mathcal{F}$ into itself, and (14) implies the continuity of the operator $Q$ in the ball $\mathcal{K}$. Thus, the operator $Q$ completely continuously transforms the ball $\mathcal{K}$ into itself. Consequently by the Schauder principle, the operator $Q$ has at least one fixed point $u(t, x)$ in the ball $\mathcal{K} \subset B_{2, T}^{1}$ By the definition of the operator $Q$ :

$$
u=Q(u) .
$$

It is clear from (9) and (10) that $u_{s}(t)=\int_{\Omega} u(t, x) v_{s}(x) d x$, the Fourier coefficients of the function $u(t, x)$ satisfy the system $(6)$ on $[0, T]$ by the system 
$\left\{v_{s}(x)\right\}_{s=1}^{\infty}$. Using this, we show that the function $u(t, x)=\sum_{s=1}^{\infty} u_{s}(t) v_{s}(x)$ is a solution almost everywhere of problem (1) - (3). It is easy to show that $u(t, x) \in \stackrel{\circ}{\mathcal{D}}_{1}\left(Q_{T}\right)$. Then, by condition 4 of the theorem, $\mathcal{F}(u(t, x)) \in \stackrel{\circ}{\mathcal{D}}_{1}\left(Q_{T}\right)$. Using this and the relation $L v_{s}(x)=-\lambda_{s}^{2} v_{s}(x)$ we transform (by integrating by parts) the system (6) to the following form:

$$
\begin{gathered}
u_{s}(t)=\varphi_{s}+\frac{1}{\lambda_{s}^{2}}\left(1-e^{-\lambda_{s}^{2} t}\right) \psi_{s}+\frac{1}{\lambda_{s}^{3}} \int_{0}^{t} \int_{\Omega}\left[\sum_{i, j=1}^{n} a_{i j}(x) \frac{\partial}{\partial x_{i}} \mathcal{F}(u(\tau, x)) \times\right. \\
\times \frac{\partial}{\partial x_{j}}\left(\frac{v_{s}(x)}{\lambda_{s}}\right)+ \\
\left.+a(x) \mathcal{F}(u(\tau, x)) \cdot \frac{v_{s}(x)}{\lambda_{s}}\right] \cdot\left[1-e^{-\lambda_{s}^{2}(t-\tau)}\right] d x d \tau, \quad s=1,2, \ldots ; t \in[0, T] .
\end{gathered}
$$

From (15), using the inequalities (8) (for the functions $\psi(x), L \varphi(x)$ ) (for the function $z(t, x)=\mathcal{F}(u(t, x))$ ), we obtain:

$$
\begin{aligned}
& \sum_{s=1}^{\infty}\left(\lambda_{s}^{3} \cdot \max _{0 \leq t \leq T}\left|u_{s}(t)\right|\right)^{2} \leq 3 \cdot\left\{\sum_{s=1}^{\infty}\left(\lambda_{s}^{3} \cdot \varphi_{s}\right)^{2}+\sum_{s=1}^{\infty}\left(\lambda_{s} \cdot \psi_{s}\right)^{2}+\right. \\
& +T \cdot \int_{0}^{T} \sum_{s=1}^{\infty}\left(\int _ { \Omega } \left[\sum_{i, j=1}^{n} a_{i j}(x) \cdot \frac{\partial F(u(\tau, x))}{\partial x_{i}} \cdot \frac{\partial}{\partial x_{j}}\left(\frac{v_{s}(x)}{\lambda_{s}}\right)+\right.\right. \\
& \left.\left.\left.+a(x) \mathcal{F}(u(\tau, x)) \cdot \frac{v_{s}(x)}{\lambda_{s}}\right] d x\right)^{2} d \tau\right\} \leq 3 \cdot\{\mathcal{D}(L \varphi(x), L \varphi(x))+ \\
& \left.+\mathcal{D}(\psi(x), \psi(x))+T \cdot \sum_{0}^{T}\left[\mathcal{D}\left(\mathcal{F}(u(\tau, x)), \frac{v_{s}(x)}{\lambda_{s}}\right)\right]^{2} d \tau\right\} \leq \\
& \leq 3 \cdot\left\{\int _ { \Omega } \left[\sum_{i, j=1}^{n} a_{i j}(x) \frac{\partial L \varphi(x)}{\partial x_{i}} \cdot \frac{\partial L \varphi(x)}{\partial x_{j}}+a(x)(L \varphi(x))^{2} d x+\right.\right. \\
& +\int_{\Omega}\left[\sum_{i, j=1}^{n} a_{i j}(x) \frac{\partial \psi(x)}{\partial x_{i}} \cdot \frac{\partial \psi(x)}{\partial x_{j}}+a(x) \psi^{2}(x)\right. \\
& \leq
\end{aligned}
$$




$$
\begin{aligned}
& +T \cdot \int_{0}^{T} \int_{\Omega}\left[\sum_{i, j=1}^{n} a_{i j}(x) \cdot \frac{\partial \mathcal{F}(u(\tau, x))}{\partial x_{i}} \cdot \frac{\partial \mathcal{F}(u(\tau, x))}{\partial x_{j}}+\right. \\
& \left.\left.+a(x)(\mathcal{F}(u(\tau, x)))^{2}\right] d x d \tau\right\} \\
& \sum_{s=1}^{\infty}\left(\lambda_{s}^{2} \cdot \max _{0 \leq t \leq T}\left|u_{s}^{\prime}(t)\right|\right)^{2} \leq 2 \cdot\left\{\sum_{s=1}^{\infty}\left(\lambda_{s}^{2} \cdot \psi_{s}\right)^{2}+\right. \\
& +\frac{1}{2} \int_{0}^{T} \sum_{s=1}^{\infty}\left(\int _ { \Omega } \left[\sum_{i, j=1}^{n} a_{i j}(x) \cdot \frac{\partial \mathcal{F}(u(\tau, x))}{\partial x_{i}} \times\right.\right. \\
& \left.\left.\left.\times \frac{\partial}{\partial x_{j}}\left(\frac{v_{s}(x)}{\lambda_{s}}\right)+a(x) \mathcal{F}(u(\tau, x)) \cdot \frac{v_{s}(x)}{\lambda_{s}}\right] d x\right)^{2} d \tau\right\} \leq \\
& \leq 2 \cdot\left\{\|L \psi(x)\|_{L_{2}(\Omega)}^{2}+\frac{1}{2} \cdot \int_{0}^{T} \sum_{s=1}^{\infty}\left[\mathcal{D}\left(\mathcal{F}(u(\tau, x)), \frac{v_{s}(x)}{\lambda_{s}}\right)\right]^{2} d \tau\right\} \leq \\
& \leq 2 \cdot\left\{\int_{\Omega}\left[\sum_{i, j=1}^{n} \frac{\partial}{\partial x_{i}}\left(a_{i j}(x) \cdot \frac{\partial \psi(x)}{\partial x_{j}}\right)-a(x) \psi(x)\right]^{2} d x+\right. \\
& +\frac{1}{2} \int_{0}^{T} \int_{\Omega}\left[\sum_{i, j=1}^{n} a_{i j}(x) \cdot \frac{\partial \mathcal{F}(u(\tau, x))}{\partial x_{i}} \cdot \frac{\partial \mathcal{F}(u(\tau, x))}{\partial x_{j}}+\right. \\
& \left.\left.+a(x)(\mathcal{F}(u(\tau, x)))^{2}\right] d x d \tau\right\} .
\end{aligned}
$$

Thus, it follows from (16) and (17) that $u(t, x) \in B_{2,2, T}^{3,2}$.

We use the following notation:

$$
u_{p, q}(t, x)=\sum_{s=p}^{q} u_{s}(t) v_{s}(x) \quad(1 \leq p \leq q) .
$$

Then, using the inequality (18) (for $k=0,1,2,3$ ) and the inequality (21) (for $r=1$ ) from [1, p. 84,88], we obtain that for any $t \in[0, T] 1 \leq p \leq q$ :

$$
\left\|u_{p, q}(t, x)\right\|_{W_{2}^{3}(\Omega)}^{2} \leq C_{1} \cdot\left\{J_{0}\left(u_{p, q}\right)+J_{1}\left(u_{p, q}\right)+J_{2}\left(u_{p, q}\right)+J_{3}\left(u_{p, q}\right)\right\} \leq
$$




$$
\begin{gathered}
\leq C_{2} \cdot\left\{J_{0}\left(u_{p, q}\right)+J_{0}\left(L u_{p, q}\right)+J_{1}\left(u_{p, q}\right)+J_{1}\left(L u_{p, q}\right)\right\}= \\
=C_{2} \cdot\left\{\int_{\Omega} u_{p, q}^{2}(t, x) d x+\int_{\Omega}\left(L u_{p, q}(t, x)\right)^{2} d x+\right. \\
+\int_{\Omega} \sum_{i, j=1}^{n} a_{i j}(x) \frac{\partial u_{p, q}(t, x)}{\partial x_{i}} \cdot \frac{\partial u_{p, q}(t, x)}{\partial x_{j}} d x+\int \sum_{\Omega}^{n} a_{i j}(x) \times \\
\left.\times \frac{\partial L u_{p, q}(t, x)}{\partial x_{i}} \cdot \frac{\partial L u_{p, q}(t, x)}{\partial x_{j}} d x\right\} \leq C_{2} \cdot\left\{\sum_{s=p}^{q} u_{s}^{2}(t)+\sum_{s=p}^{q}\left(\lambda_{s}^{2} \cdot u_{s}(t)\right)^{2}+\right. \\
\left.\left.+\sum_{s=p}^{q} \lambda_{s}^{2} \cdot u_{s}^{2}(t)+\sum_{s=p}^{q}\left(\lambda_{s}^{3} \cdot u_{s}^{(} t\right)\right)^{2}\right\} \leq \\
\leq C_{2} \cdot\left\{\frac{1}{\lambda_{1}^{6}}+\frac{1}{\lambda_{1}^{2}}+\frac{1}{\lambda_{1}^{4}}+1\right\} \cdot \sum_{s=p}^{q}\left(\lambda_{s}^{3} \cdot \max _{0 \leq t \leq T}\left|u_{s}(t)\right|\right)^{2},
\end{gathered}
$$

where $C_{1}>0, C_{2}>0$ - some constant numbers.

From (18), by virtue of the convergence of the numerical series

$$
\sum_{s=1}^{\infty}\left(\lambda_{s}^{3} \cdot \max _{0 \leq t \leq T}\left|u_{s}(t)\right|\right)^{2},
$$

it follows that $\left\|u_{p, q}(t, x)\right\|_{W_{2}^{3}(\Omega)} 0$ evenly over $t \in[0, T]$ as $p, q \rightarrow+\infty$. Consequently, the series

$$
u(t, x)=\sum_{s=1}^{\infty} u_{s}(t) v_{s}(x)
$$

and the series obtained by termwise differentiation by $x_{1}, \ldots, x_{n}$ three times converge in $L_{2}(\Omega)$ uniformly with respect to $t \in[0, T]$.

Further, using the inequality (18) (fork $=0,1,2)$ and the inequality (21) (for $r=1$ ) from [1, p. 84,88], we obtain that for any $t \in[0, T] 1 \leq p \leq q$ :

$$
\begin{gathered}
\left\|\frac{\partial u_{p, q}(t, x)}{\partial t}\right\|_{W_{2}^{2}(\Omega)}^{2} \leq C_{3} \cdot\left\{J_{0}\left(\frac{\partial u_{p, q}}{\partial t}\right)+J_{1}\left(\frac{\partial u_{p, q}}{\partial t}\right)+J_{2}\left(\frac{\partial u_{p, q}}{\partial t}\right)\right\} \leq \\
\leq C_{4} \cdot\left\{J_{0}\left(\frac{\partial u_{p, q}}{\partial t}\right)+J_{0}\left(L\left(\frac{\partial u_{p, q}}{\partial t}\right)\right)+J_{1}\left(\frac{\partial u_{p, q}}{\partial t}\right)\right\} \leq \\
\leq C_{4} \cdot\left\{\sum_{s=p}^{q}\left(u_{s}^{\prime}(t)\right)^{2}+\sum_{s=p}^{q}\left(\lambda_{s}^{2} \cdot u_{s}^{\prime}(t)\right)^{2}+\sum_{s=p}^{q}\left(\lambda_{s} \cdot u_{s}^{\prime}(t)\right)^{2}\right\} \leq
\end{gathered}
$$




$$
\leq C_{4} \cdot\left\{\frac{1}{\lambda_{1}^{4}}+1+\frac{1}{\lambda_{1}^{2}}\right\} \cdot \sum_{s=p}^{q}\left(\lambda_{s}^{2} \cdot \max _{0 \leq t \leq T}\left|u_{s}^{\prime}(t)\right|\right)^{2}
$$

where $C_{3}>0, C_{4}>0$ - some constant numbers.

From (19), in view of the convergence of the numerical series

$$
\sum_{s=1}^{\infty}\left(\lambda_{s}^{2} \cdot \max _{0 \leq t \leq T}\left|u_{s}^{\prime}(t)\right|\right)^{2}
$$

it follows that $\left\|\frac{\partial u_{p, q}(t, x)}{\partial t}\right\|_{W_{2}^{2}(\Omega)} \rightarrow 0$ evenly over $t \in[0, T]$ as $p, q \rightarrow+\infty$. Consequently, the series

$$
u_{t}(t, x)=\sum_{s=1}^{\infty} u_{s}^{\prime}(t) v_{s}(x)
$$

and the series obtained by termwise differentiation with respect to $x_{1}, \ldots, x_{n}$ twice, converge in $L_{2}(\Omega)$ uniformly with respect to $t \in[0, T]$.

As can be seen from the estimates (18) and (19):

$$
u(t, x) \in C\left([0, T] ; W_{2}^{3}(\Omega)\right), \quad u_{t}(t, x) \in C\left([0, T] ; W_{2}^{2}(\Omega)\right) .
$$

Further, it is easy to get from system (6) that $\forall t \in[0, T]$ and natural $N$, almost everywhere in $\Omega$ :

$$
\frac{\partial^{2} u_{N}(t, x)}{\partial t^{2}}-\frac{\partial}{\partial t}\left(L\left(u_{N}(t, x)\right)=\sum_{s=1}^{N}\left(\int_{\Omega} \mathcal{F}(u(t, \xi)) v_{s}(\xi) d \xi\right)_{s}(x)\right.
$$

where $u_{N}(t, x)=\sum_{s=1}^{N} u_{s}(t) v_{s}(x)$. Since $\forall t \in[0, T], \mathcal{F}(u(t, x)) \in L_{2}(\Omega)$, passing to the limit in the metric of $L_{2}(\Omega)$ in both parts of $(20)$, we obtain that $\forall t \in$ $[0, T]$ almost everywhere in $\Omega$ :

$$
\frac{\partial^{2} u(t, x)}{\partial t^{2}}-\frac{\partial}{\partial t}(L(u(t, x))=\mathcal{F}(u(t, x)) .
$$

It follows that $u_{t t}(t, x) \in L_{2}\left(Q_{T}\right)$ and the function $u(t, x)$ satisfies equation (1) almost everywhere in $Q_{T}$. And the initial conditions (2) are satisfied in an even stronger sense, namely:

$$
\|u(t, x)-\varphi(x)\|_{W_{2}^{3}(\Omega)} \rightarrow 0,\left\|u_{t}(t, x)-\psi(x)\right\|_{W_{2}^{2}(\Omega)} \rightarrow 0 \text { as } t \rightarrow+0 .
$$

Thus, the function $u(t, x)$ is a solution almost everywhere of the problem (1)-(3). The theorem is proved. 


\section{References}

[1] O.A. Ladyzhenskaya, Mixed Problem for the Hyperbolic Equation, Moscow, Gostekhizdat, 1953.

[2] E. Yukiyoshi, On some nonlinear evolution equations with the strong dissipation, Journal of Differential Equations, 45, No. 3 (1982), 332-355.

[3] T. Yu, H.-O. Yang, Initial boundary value problem for a class of strongly damped nonlinear wave equation, Journal of Harbin Engineering University, 25, No. 2 (2004), 254-256.

[4] K.I. Xudaverdiyev, S. Aliyev, A multidimensional mixed problem for one class of semilinear pseudohyperbolic equations of the third order, Baku (2012), To appear.

[5] K.I. Xudaverdiyev, A multidimensional mixed problem for nonlinear hyperbolic equations, Baku (2011), To appear. 